\title{
Early diagnosis and surgical treatment of primary cutaneous marginal zone B-cell lymphomas of the face in an Asian patient
}

Sam Yong Lee,

Woo Hyeong Kim,

Jun Ho Choi,

Kwang Seog Kim,

Jae Ha Hwang

Department of Plastic and

Reconstructive Surgery, Chonnam

National University Medical School,

Gwangju, Korea
Primary cutaneous marginal zone B-cell lymphoma (PCMZL) is an uncommon type of malignant lymphoma that mainly occurs in the trunk and upper extremity, with less frequent incidence observed on the head. Herein, we report the early diagnosis and treatment of a rare case of facial PCMZL in an Asian patient. A 51-year-old man presented with masses on the forehead and nose tip, which he had incidentally discovered 2 months previously. The masses appeared as a papule on the forehead and a patch on the nose. There were no signs of infection or bleeding, and the patient reported no other symptoms. After complete surgical excision, PCMZL was diagnosed based on the permanent biopsy. The histopathological findings revealed lymphoid aggregations with multifocal granulomas. Further treatment and follow-up were conducted at the hematology and oncology department. Despite its rarity in Asian populations, cutaneous lymphoma should be included in the differential diagnosis of facial masses. Early diagnosis and treatment based on a physical examination, imaging study, and excisional biopsy are important for a favorable prognosis.

Abbreviations: CT, computed tomography; LDH, lactate dehydrogenase; PCMZL, primary cutaneous marginal zone B-cell lymphoma; PET-CT, positron emission tomography-computed tomography.

Keywords: Asian / Face / Lymphoma

\section{INTRODUCTION}

Primary cutaneous marginal zone B-cell lymphoma (PCMZL) is a very uncommon neoplasm [1,2]. PCMZL mainly occurs in Black individuals, and Asians have a particularly low incidence $[2,3]$. The most common locations of PCMZL are the trunk and upper extremity, and it less frequently occurs on the head and neck $[1,3]$. PCMZL is often misdiagnosed due to its atypical clinical manifestations $[4,5]$. In this report, we present a rare case of PCMZL that occurred on the face of an Asian patient.

\section{Correspondence: Jae Ha Hwang}

Department of Plastic and Reconstructive Surgery, Chonnam National University

Medical School, 42 Jebong-ro, Dong-gu, Gwangju 61469, Korea

E-mail: psjhhwang@daum.net

Received September 29, 2021 / Revised October 8, 2021 / Accepted October 17, 2021

\section{CASE REPORT}

A 51-year-old man visited our hospital with skin lesions on the forehead and nose tip that he had incidentally detected 2 months ago. The patient had no other comorbidities or relevant medical history other than taking aspirin for atrial fibrillation. A physical examination demonstrated a $0.8 \times 0.8 \mathrm{~cm}$ papule at the forehead and a $2.5 \times 2.0 \mathrm{~cm}$ patch at the nose tip with erythema. No symptoms such as pain, tenderness, or bleeding were observed in either lesion. There were no masses on the other parts of the body. The C-reactive protein level was normal in the laboratory test. His recent contact history, travel history, and food history were not noteworthy. So, the possibility of infection was low.

The mass had recently exhibited rapid growth in 2 months, 
making it necessary to evaluate its potential for malignancy. Histopathological examination of a punch biopsy showed lymphoid aggregations with multiple granulomas. The tumor was therefore suspected to be a lymphoma. Complete blood count and lactate dehydrogenase (LDH) levels were normal. Contrastenhanced facial computed tomography $(\mathrm{CT})$ revealed a $0.8 \times 0.8$ $\mathrm{cm}$ mass on the forehead and a $2.5 \times 2.0 \mathrm{~cm}$ mass on the nose tip. Both lesions showed homogeneous enhancement and involvement extending from the skin to the subcutaneous layer (Fig. 1) Enlargement of lymph nodes were not seen including the neck.

Excisional surgery was done with a $3-5 \mathrm{~mm}$ safety margin. To reduce the risk of nasal asymmetry, the safety margin was set unevenly. After confirmation of tumor free margins by frozen biopsy, the defect on the nasal tip was reconstructed with a V-Y advancement flap. The forehead was covered with a local advancement flap (Fig. 2). A histopathological examination demonstrated that the forehead and nasal tumors were PCMZL
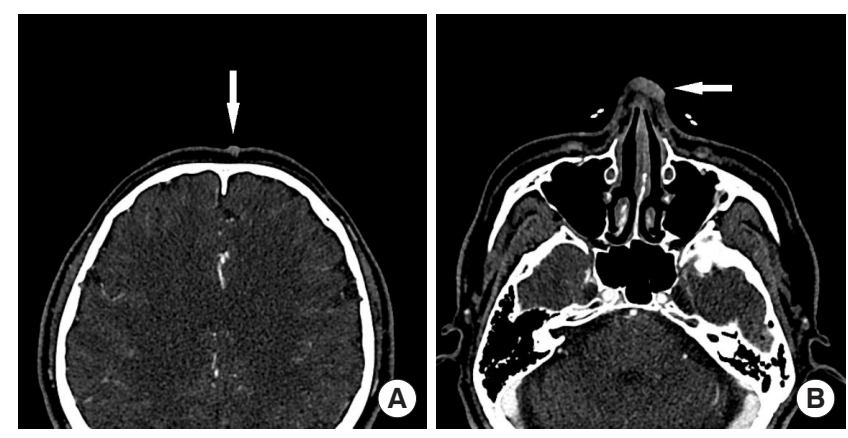

Fig. 1. A 63-year-old man with a painless soft mass on his glabellar region.
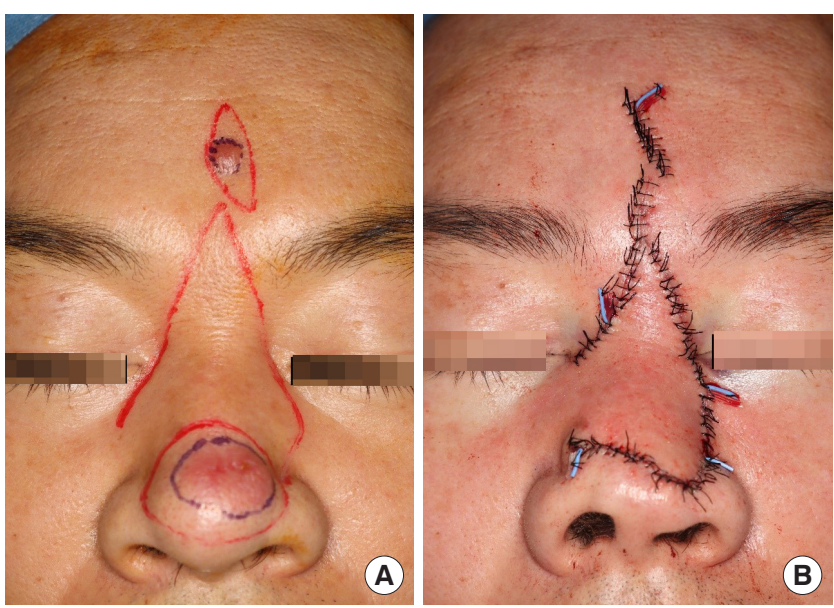

Fig. 2. The forehead defect was covered with a local advancement flap (primary closure after undermining of the wound edges) in a tension-free manner. The defect on the nasal tip was covered with a V-Y advancement flap. (A) Preoperative photograph. (B) Postoperative photograph. with lymphoid aggregations and multifocal granulomas (Fig. 3). After discharge, the patient was referred to the hemato-oncology department. Complete blood count, LDH, and beta- 2 microglobulin values were normal in follow-up laboratory tests. Positron emission tomography-computed tomography (PETCT) showed no lymph node invasion or distant metastasis (Fig. 4). No postoperative complications were seen and the surgical site was found to be well healed 6 months after the reconstruction without signs of recurrence (Fig. 5). The patient is under follow-up at the hemato-oncology department with normal laboratory tests values and without adjuvant radiotherapy or immunochemotherapy.

\section{DISCUSSION}

PCMZL is a subgroup of primary cutaneous B-cell lymphomas, which belong to the larger category of extranodal B-cell nonHodgkin lymphoma [6,7]. PCMZL accounts for about 2-7\% of

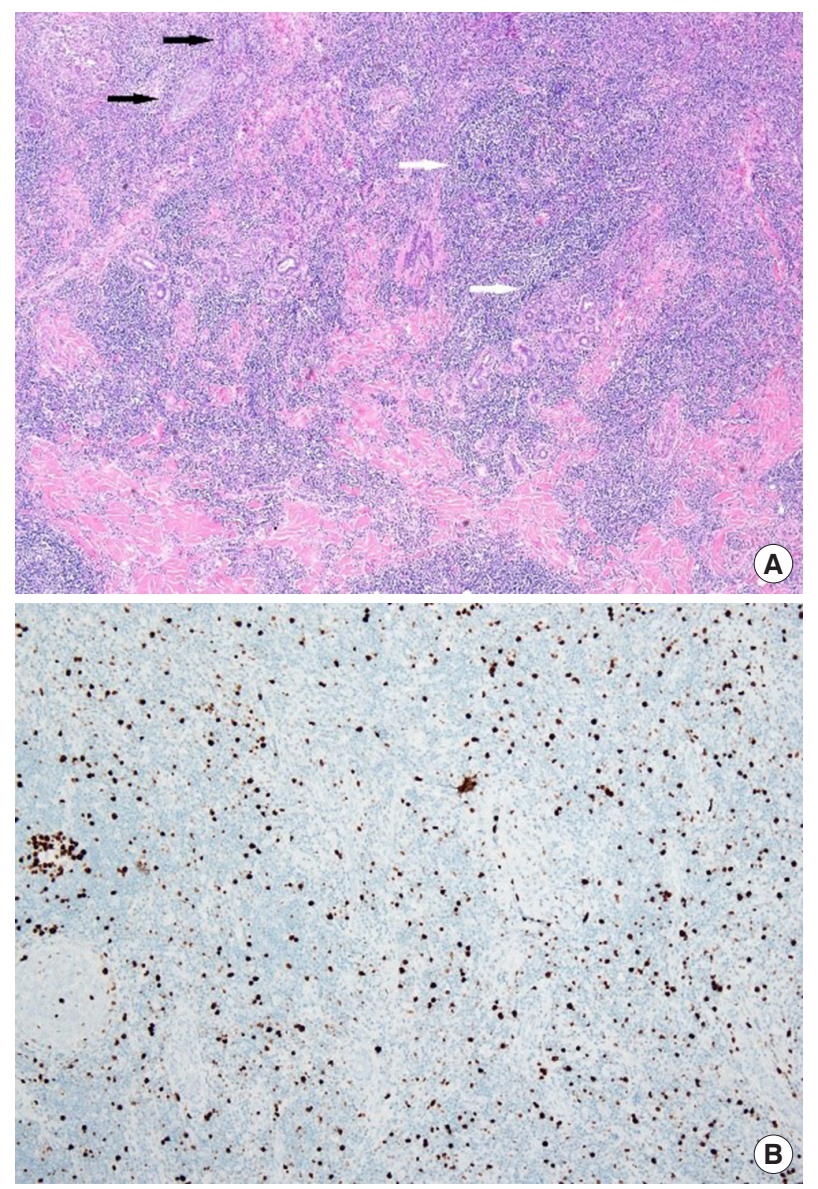

Fig. 3. Immunohistochemical examination of the biopsy specimen. (A) Lymphoid aggregations (white arrows) and multifocal granulomas (black arrows) were observed (H\&E, ×40). (B) On Ki-67 immunohistochemical staining, the specimen showed a low Ki-67 labeling index of 3\%-5\% (Ki-67 immunohistochemical staining, $\times 40$ ). 


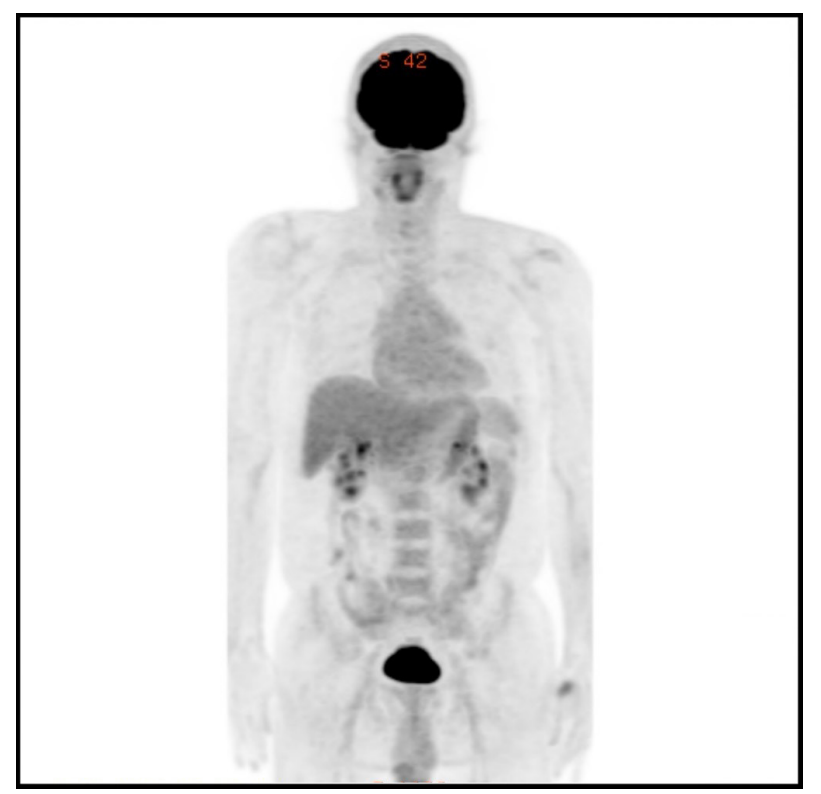

Fig. 4. Postoperative follow-up positron emission tomographycomputed tomography 1 month after the operation. There was no lymphomatous involvement suggestive of recurrence or distant metastasis.

all primary cutaneous lymphomas $[1,8]$. It appears as small, reddish-purple, single or multiple papules or nodules. PCMZL mainly occurs on the trunk and upper extremity, although it can infrequently occur on the head $[1,9]$. In this case, PCMZL presented as a papule on the forehead and a patch on the nose tip.

PCMZL most often occurs in middle-aged adults, and its incidence is higher in men than in women $[1,2]$. The prognosis of PCMZL is favorable, with a 5-year survival rate exceeding 95\% $[1,10]$. The physiopathology of PCMZL is not precisely understood, although it is thought that lymphoma develops through a reactive inflammatory lymphoproliferative process due to stimulation by chronic antigens such as viruses and bacteria [4]. Several case reports have suggested that PCMZL and Borrelia burgdorferi infection may be related, but no such cases have been reported in Asians [6]. In this case, a test for B. burgdorferi infection was not performed because South Korea is not an endemic area of this bacterium and the patient did not have any symptoms suggestive of Lyme disease. PCMZL is often asymptomatic as in this case [5]. Because of its potential for misdiagnosis as an infection or a benign mass, detailed imaging studies and laboratory tests are necessary. Above all, it is appropriate to perform a punch biopsy before excision surgery.

PCMZL is primarily diagnosed through histopathological and immunohistochemical examinations of an excisional biopsy specimen $[4,11]$. The characteristic feature of PCMZL on a histopathological examination is dermal lymphoid infiltration, which is classified as having a nodular or diffuse pattern. This

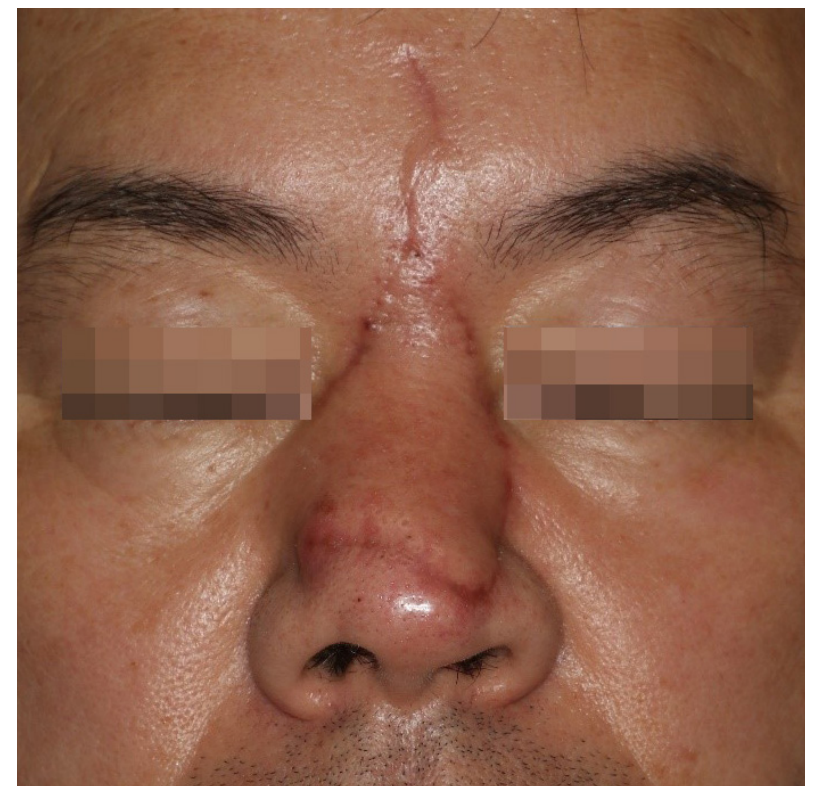

Fig. 5. Six-month follow-up photograph. There were no other signs of recurrence. Facial expression such as forehead wrinkling was normal.

infiltrate is composed of small lymphocytes, marginal zone B cells, lymphoplasmacytic cells, and plasma cells. Marginal zone B cells are surrounded by small to medium-sized cells with irregular and inconspicuous nuclei and a large amount of pale cytoplasm. On periodic acid-Schiff staining, intranuclear or intracytoplasmic inclusions can be observed, along with a predominance of lymphoplasmacytic cells $[1,6]$. A low proliferation index on Ki-67 immunohistochemical staining is another characteristic of marginal zone B-cell lymphoma [12].

PET-CT is recommended to check the degree of invasion and existence of metastasis to adjacent or distant areas [1]. In patients with PCMZL, elevated serum beta-2-microglobulin and LDH levels are sometimes observed [2]. Our patient did not show abnormally high levels of beta-2-microglobulin and LDH, but lymphoid aggregations with multifocal granulomas were observed in the histopathological examination.

For single or multiple localized lesions, surgical excision or radiotherapy is the treatment of choice [13]. Immunochemotherapy using interferon-gamma or rituximab can also be considered $[13,14]$. In the present case, all skin lesions were completely removed by surgery, and postoperative PET-CT showed no lymphoid involvement. Serum markers such as beta-2-microglobulin and LDH were also normal. Therefore, the patient is being observed without radiotherapy and immunochemotherapy.

Skin lesion recurrence occurs in approximately $50 \%$ of patients with PCMZL, but extracutaneous spread is extremely rare [1]. The diagnosis and treatment of recurrent cases follow 
the sample principles as used in primary cases. Agnihotri et al. [5] and Dumont et al. [9] recommended complete excision for solitary lesions, but did not precisely state the safety margin. In this case, wide excision of the tumor was performed, with a safety margin of 3-5 mm, to prevent recurrence and simultaneously allow the defect to be closed with a local flap. It is possible that excision with a wider safety margin can more efficiently prevent recurrence rather than simple complete excision. This hypothesis, however, needs to be verified through subsequent large-scale studies.

Although PCMZL is relatively infrequent in Asian patients and in the facial region, it must be included in the differential diagnosis of a facial mass with atypical and rapid progression. If cutaneous lymphoma is suspected, it is important to select an appropriate treatment method by testing for an infectious etiology and promptly performing an excisional biopsy to determine the cause of the lesion.

\section{NOTES}

\section{Conflict of interest}

Kwang Seog Kim and Jae Ha Hwang are editorial board members of the journal but were not involved in the peer reviewer selection, evaluation, or decision process of this article. No other potential conflicts of interest relevant to this article were reported.

\section{Ethical approval}

The study has been approved the exemption from deliberation by the Institutional Review Board of Chonnam National University Hospital (IRB No. CNUH-EXP- 2021-294).

\section{Patient consent}

The patient provided written informed consent for the publication and use of his images.

\section{ORCID}

Sam Yong Lee

Woo Hyeong Kim

Jun Ho Choi

Kwang Seog Kim

Jae Ha Hwang

https://orcid.org/0000-0002-3185-2519

https://orcid.org/0000-0003-2607-8300

https://orcid.org/0000-0002-4848-517X

https://orcid.org/0000-0002-6766-4640

https://orcid.org/0000-0001-6992-8067

\section{Author contribution}

Conceptualization: Kwang Seog Kim, Jae Ha Hwang. Data curation: Woo Hyeong Kim. Formal analysis: Woo Hyeong Kim. Methodology: Kwang Seog Kim, Jae Ha Hwang. Project administration: Jun Ho Choi. Writing - original draft: Woo Hy- eong Kim. Writing - review \& editing: Sam Yong Lee, Jun Ho Choi. Supervision: Sam Yong Lee.

\section{REFERENCES}

1. Vitiello P, Sica A, Ronchi A, Caccavale S, Franco R, Argenziano G. Primary cutaneous B-cell lymphomas: an update. Front Oncol 2020;10:651.

2. Lima M. Cutaneous primary B-cell lymphomas: from diagnosis to treatment. An Bras Dermatol 2015;90:687-706.

3. Kempf W, Kazakov DV, Buechner SA, Graf M, Zettl A, Zimmermann DR, et al. Primary cutaneous marginal zone lymphoma in children: a report of 3 cases and review of the literature. Am J Dermatopathol 2014;36:661-6.

4. Jia J, Li W, Zheng Y. Primary cutaneous diffuse large B cell lymphoma-other successfully treated by the combination of RCHOP chemotherapy and surgery: a case report and review of literature. Medicine (Baltimore) 2017;96:e6161.

5. Agnihotri T, Adotama P, Kalowitz-Bieber A, Stokar E, Meehan SA, Latkowski JA. A case of primary cutaneous marginal zone lymphoma presenting with rosacea-like eruption. JAAD Case Rep 2020;6:902-4

6. Willemze R, Jaffe ES, Burg G, Cerroni L, Berti E, Swerdlow SH, et al. WHO-EORTC classification for cutaneous lymphomas. Blood 2005;105:3768-85.

7. Baik BS, Lee WS, Ji SY, Park KS, Yang WS, Kim SY. Treatment of primary cutaneous anaplastic large cell lymphoma. Arch Craniofac Surg 2019;20:207-11.

8. Dickinson PD, Patel A, Wootton C, Bessell EM, O'Connor S. Primary cutaneous marginal zone B cell lymphoma in monozygotic twins. BMJ Case Rep 2011;2011:bcr.11.2010.3515.

9. Dumont M, Battistella M, Ram-Wolff C, Bagot M, de Masson A. Diagnosis and treatment of primary cutaneous B-cell lymphomas: state of the art and perspectives. Cancers (Basel) 2020; 12:1497.

10. Lang CCV, Ramelyte E, Dummer R. Innovative therapeutic approaches in primary cutaneous B cell lymphoma. Front Oncol 2020;10:1163.

11. Kim J, Jeong M, Jun D, Lee M, Shin D, Kim W, et al. Primary cutaneous CD4+ small/medium T-cell lymphoma: a case report. Arch Craniofac Surg 2021;22:199-203.

12. Lee CL, Lee IS, Jung SG. A case of nodal marginal zone B-cell lymphoma of the lower eyelid. Arch Craniofac Surg 2020;21: 249-52.

13. Selva R, Violetti SA, Delfino C, Grandi V, Cicchelli S, Tomasini $\mathrm{C}$, et al. A Literature revision in primary cutaneous B-cell lymphoma. Indian J Dermatol 2017;62:146-57.

14. Penate Y, Hernandez-Machin B, Perez-Mendez LI, Santiago F, 
Rosales B, Servitje O, et al. Intralesional rituximab in the treatment of indolent primary cutaneous B-cell lymphomas: an epidemiological observational multicentre study. The Spanish
Working Group on Cutaneous Lymphoma. Br J Dermatol 2012;167:174-9. 